\author{
Marc Leone \\ Carole Bechis \\ Karine Baumstarck
}

\section{De-escalation in severe sepsis: still an important part of our armamentarium against antimicrobial resistance, of course!}

Accepted: 28 August 2014

Published online: 19 September 2014

(C) Springer-Verlag Berlin Heidelberg and ESICM 2014

Dear Editor,

We thank Kapoor and Saigal [1] for their relevant comments about our study on de-escalation versus continuation of empirical antimicrobial treatment in severe sepsis [2]. We agree that de-escalation cannot be discarded on the basis of a single randomized clinical trial. Our manuscript does not convey this message. However, this first randomized clinical trial is probably a red flag that must drive us to better define conditions with which de-escalation can be safely performed.

In agreement with observational studies [3], our trial in patients with severe sepsis showed that de-escalation did not affect mortality. In contrast, de-escalation was associated with an increased use of antimicrobials. This finding was reported in a recent cohort of septic patients with neutropenia [4]. Our study suggests that this was related to an increased number of superinfections in the deescalation group. The duration of intensive care unit stay was also increased in the de-escalation group. In our opinion, these surprising results were never properly addressed in previous observational studies. In those studies, de-escalation was probably performed in patients with an adequate response to antibiotics.

This is a major flaw in order to assess the risk associated with this strategy [5].

We strongly agree that there is a need for a consensual definition of deescalation. In our pragmatic approach, we did not take into account the specific minimum inhibitory concentration of each organism. That is probably a limitation in our study. However, to our knowledge, there is no evidence showing that a strategy of antibiotic stewardship based on the minimum inhibitory concentration would improve the patient outcomes.

Our trial attempted to reflect reallife practices. In routine, de-escalation is used in both surgical and medical patients. We agree that deescalation is probably associated with a higher risk of harm in patients with suspected polymicrobial infection, such as peritonitis, than in those with positive blood culture due to catheterrelated infection. To our knowledge, there is no recommendation for a specific strategy of antimicrobial stewardship depending on those features. We hope that our results pave the way for future studies on antibiotic stewardship in the intensive care unit.

Conflicts of interest Dr. Leone reported serving as a consultant for LFB. No other disclosures were reported.

\section{References}

1. Kapoor G, Saigal S (2014) De-escalation in severe sepsis: still an important part of our armamentarium against antimicrobial resistance. Intensive Care Med. doi: 10.1007/s00134-014-3476-4
2. Leone $\mathrm{M}$, Bechis $\mathrm{C}$, Baumstarck $\mathrm{K}$, Lefrant JY, Albanèse J, Jaber S, Lepape A, Constantin JM, Papazian L, Bruder N, Allaouchiche B, Bézulier K, Antonini F, Textoris J, Martin C, for the AZUREA Network Investigators (2014) Deescalation versus continuation of empirical antimicrobial treatment in severe sepsis: a multicenter non-blinded randomized noninferiority trial. Intensive Care Med. doi: 10.1007/s00134-014-3411-8

3. Leone M, Bourgoin A, Cambon S, Dubuc M, Albanèse J, Martin C (2003) Empirical antimicrobial therapy of septic shock patients: adequacy and impact on the outcome. Crit Care Med 31:462-467

4. Mokart D, Slehofer G, Lambert J, Sannini A, Chow-Chine L, Brun JP, Berger P, Duran S, Faucher M, Blache JL, Saillard C, Vey N, Leone M (2014) De-escalation of antimicrobial treatment in neutropenic patients with severe sepsis: results from an observational study. Intensive Care Med 40:41-49

5. Garnacho-Montero J, GutiérrezPizarraya A, Escoresca-Ortega A, Corcia-Palomo Y, Fernández-Delgado E, Herrera-Melero I, Ortiz-Leyba C, Márquez-Vácaro JA (2014) Deescalation of empirical therapy is associated with lower mortality in patients with severe sepsis and septic shock. Intensive Care Med 40:32-40

\section{Leone (®) - C. Bechis}

Service d'Anesthésie et de Réanimation, Hôpital Nord, Aix Marseille Université, 13015 Marseille, France

e-mail: marc.leone@ap-hm.fr

Tel.: +33491968655

K. Baumstarck

Unité d'Aide Méthodologique à la

Recherche Clinique et Epidémiologique, Aix Marseille Université, Marseille, France 